\title{
Evidence for Altered Epicardial Coronary Artery Autoregulation as a Cause of Distal Coronary Vasoconstriction after Successful Percutaneous Transluminal Coronary Angioplasty
}

Tim A. Fischell, Kurt N. Bausback, and Thomas V. McDonald

Division of Cardiology, Stanford University Medical Center, Stanford, California 94305

\begin{abstract}
To determine whether vasoconstriction distal to the site of successful percutaneous transluminal coronary angioplasty (PTCA) is a result of altered autoregulation in a hypoperfused coronary artery, we examined the association of this distal vasoconstriction with lesion severity in 20 patients. Lesion severity was classified as moderate, severe or critical $(>1.0$, $0.5-1.0$, and $<0.5 \mathrm{~mm}$, respectively). Quantitative coronary measurements were made at 3,15 , and $30 \mathrm{~min}$ after PTCA, and then after intracoronary (IC) nitroglycerin, in coronary segments distal to the dilated lesion (distal) and in a nonmanipulated vessel (control).

Coronary vasoconstriction in the Distal segment after PTCA correlated with lesion severity, with $14 \pm 4 \%, 28 \pm 2 \%$, and $41 \pm 5 \%$ vasoconstriction (vs. IC nitroglycerin, $30 \mathrm{~min}$ after PTCA) in the moderate, severe and critical lesion severity subgroups, respectively $(P<0.01$ for critical or severe vs. moderate). This vasoconstriction was significantly greater than that observed in the corresponding control segment for patients with severe $(P<.0 .01)$, and critical $(P<0.001)$ lesions.

These findings suggest that hypoperfused human epicardial coronary arteries "reset" their autoregulatory responsiveness and that distal vasoconstriction after PTCA is the result of this altered autoregulation. These findings have clinical implications concerning the etiology, prophylaxis and treatment of coronary spasm after PTCA and coronary bypass surgery. (J. Clin. Invest. 1990. 86:575-584.) Key words: epicardial • coronary artery $\bullet$ vasoconstriction $\bullet$ angioplasty $\bullet$ autoregulation
\end{abstract}

\section{Introduction}

In 1902 Bayliss first described the observation that rapid increases in intraluminal arterial pressure were capable of provoking profound vasoconstriction in both denervated arteries in vivo, and in excised arterial segments (1). This phenomenon was attributed to a stretch-dependent reflex of arterial smooth muscle, and has been elaborated as the "myogenic hypothesis" of arterial tone regulation $(2,3)$. In the dog a rapid increase in blood pressure induced by methoxamine initially causes epi-

Address reprint requests to Dr. Fischell, Falk Cardiovascular Research Center, Stanford University, Medical Center, 300 Pasteur Drive, Stanford, CA 94305.

Received for publication 25 September 1989 and in revised form 13 March 1990.

J. Clin. Invest.

(C) The American Society for Clinical Investigation, Inc.

$0021-9738 / 90 / 08 / 0575 / 10 \quad \$ 2.00$

Volume 86, August 1990, 575-584 cardial coronary artery dilation (stretch) followed by severe vasoconstriction (4). Although these data have been interpreted as $\alpha$ adrenergic mediated vasoconstriction, the time course of this response may be more consistent with a stretch/ pressure-dependent form of epicardial coronary vasoconstriction. The importance of this myogenic reflex in the autoregulation of human epicardial coronary artery tone is suggested by the finding that coronary tone is essentially independent of autonomic or humoral input (5). Recent observations have suggested that chronic hypoperfusion may predispose to severe arterial spasm after bypass grafting of totally occluded coronary arteries (6). Given these observations, we hypothesized that the distal vasoconstriction which has been observed after percutaneous transluminal coronary angioplasty (PTCA) ${ }^{1}$ (7) may result from a rapid post-PTCA increase in perfusion pressure causing pressure/stretch-dependent vasoconstriction in hypoperfused epicardial coronary arteries with altered (reset) autoregulation. If this hypothesis were correct one would expect to see much greater distal vasoconstriction after successful dilatation of a critical/subtotal stenosis with its large associated resting transstenotic pressure gradient (hypoperfused) than after dilatation of a moderate lesion with virtually no associated transstenotic pressure gradient at physiologic coronary blood flow(s) (not hypoperfused) (8).

The purpose of this study was to test this hypothesis by examining whether or not the severity of this post-PTCA distal vasoconstriction was correlated with lesion severity and/or qualitative assessment of coronary blood flow before PTCA. Accordingly, we prospectively studied, using quantitative arteriography, the spontaneous post-PTCA vasoconstrictor responses in the coronary segment distal to the dilated lesion and in a nonmanipulated (control) segment in 20 patients with varying lesion severity who underwent uncomplicated single vessel PTCA.

\section{Methods}

Patients. 20 patients scheduled for elective single vessel PTCA of focal stenoses were prospectively entered into the study. All patients had angina pectoris despite medical therapy. Exclusion criteria included concurrent nitroglycerin therapy, recent $(<2 \mathrm{wk})$ myocardial infarction, length of lesion to be dilated greater than $10 \mathrm{~mm}$, grade 3 collateral filling of the target vessel before PTCA $(9,10)$, requirement for intracoronary nitroglycerin during the PTCA, greater than $50 \%$ residual stenosis and/or less than thrombolysis in myocardial infarction (TIMI) grade 3 coronary blood flow (11) after PTCA, refusal to participate in the study and/or technically inadequate coronary arteriography. Attempts were made to enroll patients with left coronary artery

1. Abbreviations used in this paper: CSA, cross-sectional area; EDRF, endothelium-derived relaxing factor; IC, intracoronary; PTCA, percutaneous transluminal coronary angioplasty; TIMI, thrombolysis in myocardial infarction. 
lesions so that the nonmanipulated left coronary vessel (e.g., left circumflex when left anterior descending was dilated) could be used for control measurements.

Procedure. The basic protocol for study has been described in detail previously (7). All patients agreed to proceed with the PTCA and study procedures after being informed of the potential risks and complications, as outlined in a protocol approved by the Institutional Review Board of the Stanford University Medical Center. All patients took their usual oral medications including aspirin and calcium channel blockers on the day of the procedure. After premedication with diazepam and local anesthesia with $0.25 \%$ bupivicaine femoral arterial $(8$ Fr) and femoral venous ( $7 \mathrm{Fr}$ ) sheaths were placed via single wall entry technique. $5000 \mathrm{U}$ i.v. of heparin was given. In those patients undergoing PTCA of the right coronary artery, a bipolar temporary pacing catheter was placed in the right ventricular apex. Selective coronary arteriography of the vessel to be dilated was performed in multiple projections, including at least one pair of orthogonal views, using the 4-5-in image CsI intensifier mode and Renografin $76^{R}$ at a cine rate of 30 frames per s. The precise angle of each projection was recorded so that the projection that best demonstrated the stenosis to be dilated with minimal foreshortening could be replicated. All contrast injections throughout a given study were performed by the same operator in order to minimize variability in angiographic technique.

After a second bolus of 5,000 U i.v. of heparin coronary angioplasty was performed using either an over the wire balloon catheter system (16 patients) or a fixed wire balloon catheter system (4 patients). Balloon sizes were chosen to approximate the diameter of the "normal" coronary segment adjacent to the segment to be dilated. At least two balloon inflations were performed in each case, with additional inflations performed as needed until the coronary stenosis had been dilated adequately using angiographic and/or hemodynamic criteria.

As soon as was feasible after the final balloon inflation (average 3 min, range 2-5 $\mathrm{min}$ ) the balloon catheter and guidewire were withdrawn and selective coronary arteriography, in the previously selected projection that best demonstrated the stenosis, was performed. This angiogram was designated post-PTCA. The coronary guiding catheter was then withdrawn and replaced with an $8 \mathrm{Fr}$ right or left coronary artery Judkin's "marker" catheter, to be used as a reference for coronary quantification (see below). Coronary arteriograms (same projection) were repeated at 15 and $30 \mathrm{~min}$ after final balloon inflation, and again $3 \mathrm{~min}$ after the administration of $300 \mu \mathrm{g}$ of IC nitroglycerin given immediately after the 30 -min angiogram ( $33 \mathrm{~min}$ after final balloon inflation). This dose of IC nitroglycerin was chosen to provide a near maximal vasodilating effect. Blood pressure and heart rates were recorded immediately preceding each arteriogram (pre-PTCA, postPTCA, $15 \mathrm{~min}, 30 \mathrm{~min}$, after IC nitroglycerin).

Coronary arteriographic analysis. All films were analyzed by quantitative arteriographic techniques. This system utilizes automated computer assisted edge detection of a digitized cineangiographic image, as has been described in detail in prior reports $(7,12)$. Wellopacified end-diastolic cine frames were selected as described, and magnified $(\times 3.5)$ with the appropriate coronary segment centered in the image field. The image was digitized (model 5524; DeAnza Systems, Fremont, CA) with the video processor controlled by an 2100 computer (Hewlett Packard, Palo Alto, CA). The digitized image was displayed on a graphics computer terminal linked to a light pen. Magnification correction was achieved using a cylindrical tantalum marker of known diameter that is attached to the tip of the coronary catheter in the field of view. After the segment length was designated on the computer terminal, the light pen was used to manually trace the margins of the appropriate vessel segment, and to indicate the fiducial point. The maximum derivative of the density profile perpendicular to these manually defined margins are defined as the computer generated vascular boundaries. Using these computer generated vascular boundaries, the minimum, maximum, and mean diameters of the vessel segment were calculated. The resolution of this system has been demonstrated to be $\pm 0.06 \mathrm{~mm}$ (12).

Three coronary segments were analyzed in patients undergoing PTCA in the left coronary artery system, as illustrated in Fig. $1 \mathrm{~A}$. Segment 1 (Distal segment) was chosen to be a clearly identifiable 5-mm long segment distal to the dilated segment and not manipulated by the balloon catheter. Segment 2 (Angioplasty segment) was defined as the $5-\mathrm{mm}$ long segment centered on the narrowest point of the coronary stenosis as viewed in the pre-PTCA arteriogram. Segment 3 (Control segment) was defined as a clearly identifiable 5-mm long segment in the left coronary artery that was not manipulated by guide wire or balloon catheter (e.g., left circumflex when PTCA was performed in the left anterior descending coronary artery). For the three patients undergoing right coronary artery PTCA, only segments 1 and 2 could be analyzed since there was no equivalent Control segment (see Fig. $1 B$ ).

One of the two designated film readers analyzed the mean and minimum segmental diameters in each of three consecutive end-diastolic frames (marked automatically on the cine film by an ECG marker) for each 5-mm long segment, at each time/condition. The final segmental vessel diameter (mean and minimum) at each time was defined as the mean of the three end-diastolic measurements. Based upon measurements of minimal lesion diameter using quantitative coronary arteriography in orthogonal projections prior to PTCA, the patients were divided into moderate $(n=7)$, severe $(n=6)$, and critical $(n=7)$ lesion severity subgroups (minimal lesion diameters of $>1.0$, $0.5-1.0$, and $<0.5 \mathrm{~mm}$, respectively). Minimal lesion cross-sectional area (CSA) was calculated assuming an elliptical model where D1 $=$ minimal lesion diameter in one orthogonal projection, and D2 = minimal lesion diameter in the second orthogonal projection (13): $\mathrm{CSA}=\pi(\mathrm{D} 1 \times \mathrm{D} 2) / 4$. For the purposes of determining percent $(\%)$

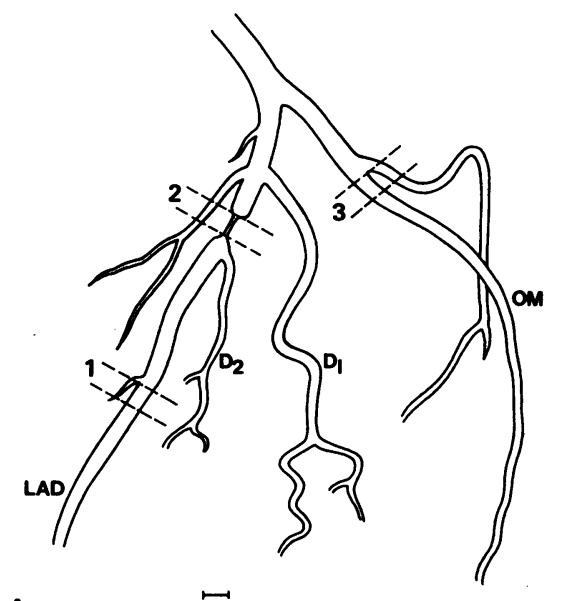

A

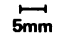

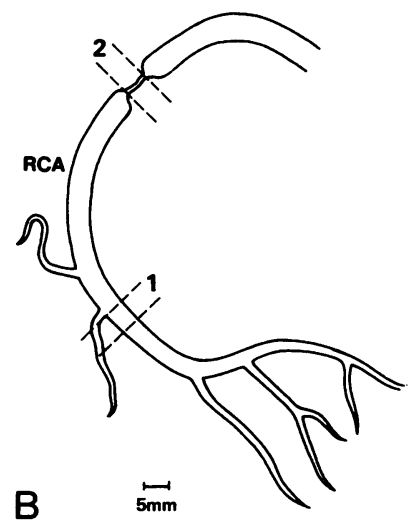

Figure 1. Diagram of coronary artery segments analyzed by quantitative coronary arteriography in the left $(A)$ and right $(B)$ coronary arteries. Segment 1 (Distal segment) was defined as a clearly identifiable 5-mm long segment distal to the dilated lesion. Segment 2 (Angioplasty segment) was defined as a 5-mm long segment centered in the narrowest point of the coronary stenosis to be dilated. Segment 3 (Control segment) was defined as a 5-mm long segment in the left coronary artery not manipulated by the guidewire or balloon catheter. OM, obtuse marginal branch; D1 and D2, first and second diagonal branches, respectively. (Reproduced from Circulation. 1988. 78(6):1323-1334, with permission from the American Heart Association.) 
vasoconstriction, the segmental vessel diameters measured from the post-IC nitroglycerin angiogram were defined as the maximally vasodilated state such that: \% Vasoconstriction $(t)=$ Diameter after NTG - Diameter at Time $t /$ Diameter after nitroglycerin.

The coronary blood flow of the treated vessel in the pre-PTCA and immediate post-PTCA arteriograms was graded according to TIMI classification (i.e., grades $0-3$ ) (11) by two film readers before quantitative arteriographic analysis of the films. Coronary collateral flow before PTCA was graded on a 0-3 point scale as described by Rentrop (9) and Fujita (10).

Statistics. Data are presented as the mean \pm standard error (SEM) unless otherwise stated. The comparisons of percent vasoconstriction between segments at a given time were performed using a Student's paired $t$ test. Comparisons of percent vasoconstriction in each segment for each condition in the various lesion severity subgroups, and patient and procedural variables were analyzed by a one-way analysis of variance, using repeated measures (Fisher PLSD). Analysis of scattergram plots of percent vasoconstriction vs. minimal lesion diameter and cross-sectional area was performed using a polynomial regression approach. A $P$ value of $<0.05$ was considered statistically significant.

Drugs. Nitroglycerin for intracoronary and intravenous administration was prepared by the addition of $25 \mathrm{mg}$ of nitroglycerin (Tridil ${ }^{\mathrm{R}}$, DuPont Pharmaceuticals, Wilmington, DE) to $250 \mathrm{ml}$ of normal saline, yielding a final concentration of $100 \mu \mathrm{g} / \mathrm{ml}$. Immediately after the 30-min arteriogram, $3 \mathrm{ml}$ of this solution was administered as an intracoronary injection via the right or left coronary artery diagnostic catheter.

\section{Results}

The patient and PTCA procedural data $(n=20)$ are shown in Table I for patients in each of the lesion severity subgroups (moderate [ $n=7]$, severe [ $n=6]$, and critical [ $n=7]$ ). There were no significant differences in the historical variables between the groups. 16 of the 20 patients were taking orally administered calcium channel blockers (either diltiazem [ $n$ $=12$ ] or nifedipine [ $n=4]$ ), and 15 of 20 patients were treated with aspirin $(>2 \mathrm{wk})$ at the time of entry into the study (see Table I). All patients received their usual oral doses of these medications before their PTCA. There were no statistically significant differences between groups in the type or dosage of medications administered before the study.

The critical lesion severity subgroup had significantly lower TIMI flow prior to PTCA than either the severe or moderate lesion severity subgroups (Table I). The lesion severity before PTCA, for each subgroup, is also shown in Table $I$. Primary angiographic success, defined as a reduction in stenosis diameter to less than $50 \%$ and TIMI grade 3 flow, was achieved in all 20 patients. There was no significant difference in the post-PTCA luminal diameters in the dilated segment between the critical, severe and moderate lesion subgroups (Table I). There was a modest and nearly equal reduction in systolic blood pressure after intracoronary nitroglycerin in all three subgroups $(12,10$, and $10 \mathrm{mmHg}$ reductions in the moderate, severe, and critical lesion subgroups, respectively; $P$ $<0.05$ vs. 30 min after PTCA) without any significant change in blood pressure from before PTCA to $30 \mathrm{~min}$ after PTCA in any group. There were no statistically significant differences in systolic, diastolic or mean blood pressures between subgroups at any time. There were no major complications associated with any of these procedures (i.e., no MI, death, or need for coronary artery bypass surgery).

Table I. Patient and Procedural Data

\begin{tabular}{|c|c|c|c|}
\hline Lesion severity: & Moderate (> $1.0 \mathrm{~mm})$ & Severe $(0.5-1.0 \mathrm{~mm})$ & Critical $(<0.5 \mathrm{~mm})$ \\
\hline Patients $(n)$ & 7 & 6 & 7 \\
\hline Age (mean \pm SEM, $y r$ ), gender & $57 \pm 1.9,7$ male & $61 \pm 5.4,5$ male, 1 female & $61 \pm 1.6,7$ male \\
\hline Vessel dilated & $2 \mathrm{LAD}, 4 \mathrm{Cx} ., 1 \mathrm{RCA}$ & 3 LAD, 2 Cx., 1 RCA & $4 \mathrm{LAD}, 2 \mathrm{Cx} ., 1 \mathrm{RCA}$ \\
\hline Balloon size (mean, $\mathrm{mm}$ ) & 2.8 & 2.7 & 2.8 \\
\hline Balloon size: reference seg. (mean \pm SEM) & $1.06 \pm 0.07$ & $0.96 \pm 0.10$ & $0.93 \pm 0.12$ \\
\hline Number of inflations (mean, $n$ ) & 3.2 & 5.0 & 4.4 \\
\hline Maximal inflation pressure (mean, atm) & 7.3 & 7.8 & 7.8 \\
\hline TIMI flow pre-PTCA (mean \pm SEM) & $3 \pm 0.0$ & $2.3 \pm 0.4$ & $1.3 \pm 0.3^{*}$ \\
\hline Grade $1(n)$ & $0 / 7$ & $1 / 6$ & $5 / 7$ \\
\hline Grade $2(n)$ & $0 / 7$ & $1 / 6$ & $2 / 7$ \\
\hline Grade $3(n)$ & $7 / 7$ & $4 / 6$ & $0 / 7$ \\
\hline \multicolumn{4}{|l|}{ Lesion diameter } \\
\hline pre-PTCA (mean \pm SEM, $\mathbf{m m}$ ) & $1.31 \pm 0.11$ & $0.64 \pm .04 \ddagger$ & $0.43 \pm 0.01^{*}$ \\
\hline post-PTCA (mean \pm SEM, $\mathrm{mm}$ ) & $2.16 \pm 0.23$ & $1.91 \pm 0.32$ & $1.82 \pm 0.25$ \\
\hline \multicolumn{4}{|l|}{ Distal segment diameter } \\
\hline After nitroglycerin (mean \pm SEM, $m m$ ) & $2.28 \pm 0.20$ & $2.01 \pm .08$ & $2.04 \pm .23$ \\
\hline \multicolumn{4}{|l|}{ Medications: } \\
\hline Diltiazem & $3 / 7$ patients & $4 / 6$ patients & $5 / 7$ patients \\
\hline Nifedipine & $2 / 7$ patients & $1 / 6$ patients & $1 / 7$ patients \\
\hline Aspirin & $4 / 7$ patients & $6 / 6$ patients & $5 / 7$ patients \\
\hline Dipyridamole & $1 / 7$ patients & 1/6 patients & $0 / 7$ patients \\
\hline Beta blockers & $1 / 7$ patients & $1 / 6$ patients & 2/7 patients \\
\hline
\end{tabular}

Cx., left circumflex artery; LAD, left anterior descending coronary artery; RCA, right coronary artery; ${ }^{*} P<0.001$ for critical vs. severe and moderate subgroups. $\ddagger P<0.001$ for severe vs. moderate subgroup. 
Distal coronary vasoconstriction after PTCA. The severity of vasoconstriction in the Distal versus the Control segment at $30 \mathrm{~min}$ after successful PTCA for patients in the moderate, severe and critical lesion severity subgroups is illustrated in Fig. $2 A$. As shown, the degree of vasoconstriction was closely associated with lesion severity before PTCA. There was no significant difference in percent vasoconstriction (at $30 \mathrm{~min}$ ) between the Control vs. Distal segment vasoconstriction in the moderate lesion subgroup ( $12 \pm 1 \%$ vs. $14 \pm 2 \%$, respectively, $P$ $=\mathrm{NS})$. The severe and critical lesion subgroups demonstrated significantly greater distal vasoconstriction than the moderate lesion subgroup ( $28 \pm 2 \%$ and $41 \pm 5 \%$ vs. $14 \pm 2 \%$, respectively, $P$ values as shown). Similarly (Fig. $2 B$ ) there was significantly greater vasoconstriction in the Distal segment in those patients with impaired translesional blood flow before PTCA (TIMI grade $<3$ subgroup, $n=10$ ) than in those with qualitatively normal epicardial blood flow (TIMI grade 3 subgroup, $n=10$ ) ( $36 \pm 3 \%$ vs. $16 \pm 2 \%$, respectively, $P<0.005$ ). In Fig. 3 , percent vasoconstriction at $30 \mathrm{~min}$ after PTCA is plotted as a function of minimal lesion diameter $(A, C$, and $E)$, and lesion crosssectional area $(B, D$, and $F)$ before PTCA for the Distal, Angioplasty, and Control segments. Using a polynomial regression analysis of the scattergram plots, there was a significant correlation $(P<0.001)$ between lesion severity, as judged by either minimal lesion diameter or cross-sectional area, and percent vasoconstriction for the Distal segment. In contrast, there was no association between lesion severity and postPTCA vasoconstriction in the Control segment or in the Angioplasty segment $\left(R^{2}=0.012, R^{2}=0.011, P=\mathrm{NS}\right.$, for Control and Angioplasty segments, respectively). Fig. 4 shows an example of severe post-PTCA vasoconstriction observed distal to a subtotal occlusion compared to the modest and stable degree of coronary tone in the nonmanipulated left coronary artery (Control) of the same patient.

Time course of distal vasoconstriction. The time course of the distal vasoconstriction observed in the severe and critical lesion severity subgroups (grouped together as Distal segment (s), $n=13$ ) is illustrated in Fig. 5. The Distal segment in both the severe/critical and the moderate lesion severity (distal segment $(\mathrm{m}), n=7)$ groups demonstrated normal "resting" tone before PTCA ( $10 \pm 2 \%$ and $12 \pm 2 \%$, respectively). There was a minor increase in Distal vasoconstriction noted in the imme- diate post-PTCA angiogram in the severe/critical group but not in the moderate lesion ( $14 \pm 3 \%$ vs. $8 \pm 3 \%$, respectively). At 15 and $30 \mathrm{~min}$ after PTCA there was marked vasoconstriction in the Distal segment of the severe/critical group but not in the moderate lesion or Control groups $(31 \pm 4 \%$ and $33 \pm 3 \%$, vs. $12 \pm 2 \%$ and $14 \pm 2 \%$, and $14 \pm 2 \%$ and $12 \pm 1 \%$ for the severe/ critical, moderate and Control, respectively). The increase in percent vasoconstriction from pre-PTCA to $30 \mathrm{~min}$ after PTCA was also significantly greater for the severe $(21 \pm 3 \%)$ and critical $(27 \pm 6 \%)$ lesion severity subgroups than for the moderate $(2 \pm 2 \%)$ lesion group ( $P$ values $<0.001$ for severe or critical vs. moderate). These data suggest that the maximal vasoconstriction in the Distal segment occurs within 15-30 min after successful PTCA.

Vasoconstriction in angioplasty segment. As previously reported (7) we again observed progressive vasoconstriction in the dilated (Angioplasty) segment after PTCA with $2 \pm 2 \%$, $24 \pm 2 \%$ and $28 \pm 1 \%$ vasoconstriction (vs. IC nitroglycerin diameter) at 3 (post-PTCA), 15, and $30 \mathrm{~min}$ after PTCA, respectively (Fig. 5). The degree of vasoconstriction observed 15 and 30 min after PTCA in the dilated segment was significantly greater than that seen immediately post-PTCA $(P<0.0001)$, and was significantly greater than the vasoconstriction of the Control segment at 15 and $30 \mathrm{~min}(P$ values $<0.005)$.

\section{Discussion}

Coronary artery vasoconstriction distal to the site of balloon dilatation has been observed after successful PTCA, and has been previously attributed to the release of platelet-derived vasoactive substances at the proximal site of balloon-induced arterial injury (7). This study demonstrates that the severity of this distal vasoconstriction is significantly correlated with lesion severity before PTCA. These findings suggest that human epicardial coronary arteries reset their autoregulatory response in the setting of chronic hypoperfusion and that this distal vasoconstriction is the result of a maladaptive constrictor response to increased perfusion pressure after successful PTCA.

The regulation of arterial smooth muscle tone is complex and involves an interplay between humoral, neurogenic, myogenic, and endothelium-derived factors (14). After successful PTCA the distal coronary artery bed may be subjected to a
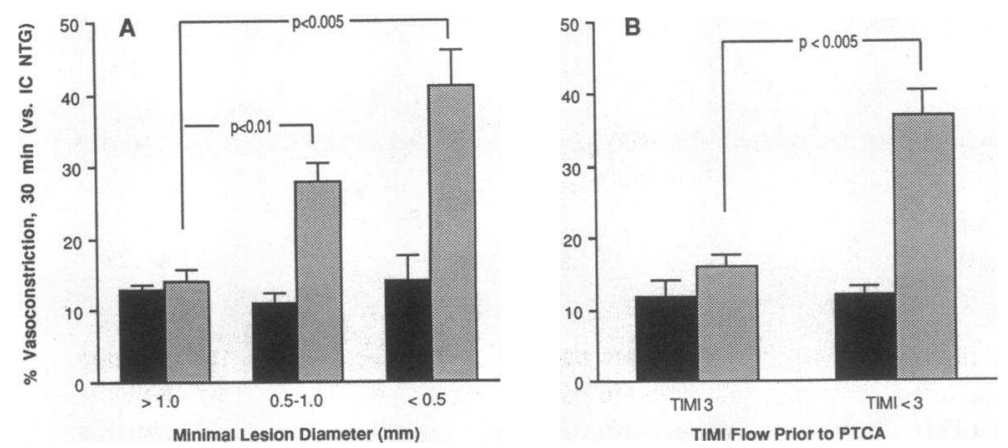

Figure 2. Bar graphs demonstrating the degree of vasoconstriction in the Control (black) and Distal (stippled) segments 30 min after PTCA as a function of lesion severity $(A)$ and TIMI flow prior to PTCA $(B)$. Vertical bars represent percent vasoconstriction (mean \pm SEM, vs. intracoronary, IC, nitroglycerin diameter, see text). $(A)$ Percent vasoconstriction at $30 \mathrm{~min}$ after PTCA is shown for the Distal and Control segments as a function of lesion severity for patients with minimal lesion diameters of $>1.0$ (moderate lesion subgroup, $n=6$ ), $0.5-1.0 \mathrm{~mm}$ (severe lesion subgroup, $n=6$ ), and $<0.5 \mathrm{~mm}$ (critical lesion subgroup, $n=7$ ) ( $P$ values as shown). There was no significant difference in vasoconstriction in the Control segments between the three groups nor between the Control and Distal segment for the moderate lesion subgroup. $(B)$ Percent vasoconstriction at $\mathbf{3 0}$ min after PTCA is shown for the Distal and Control segments for patients with normal coronary blood flow ("TIMI $3, " n=10$ ) and for patients with impaired coronary blood flow ("TIMI $<3$," $n=10$ ) prior to PTCA. There was significantly greater vasoconstriction in the Distal segment for the subgroup with impaired coronary blood flow compared to the subgroup with normal flow ( $P$ value as shown). There was no significant difference in vasoconstriction in the Control segments in the two groups. 

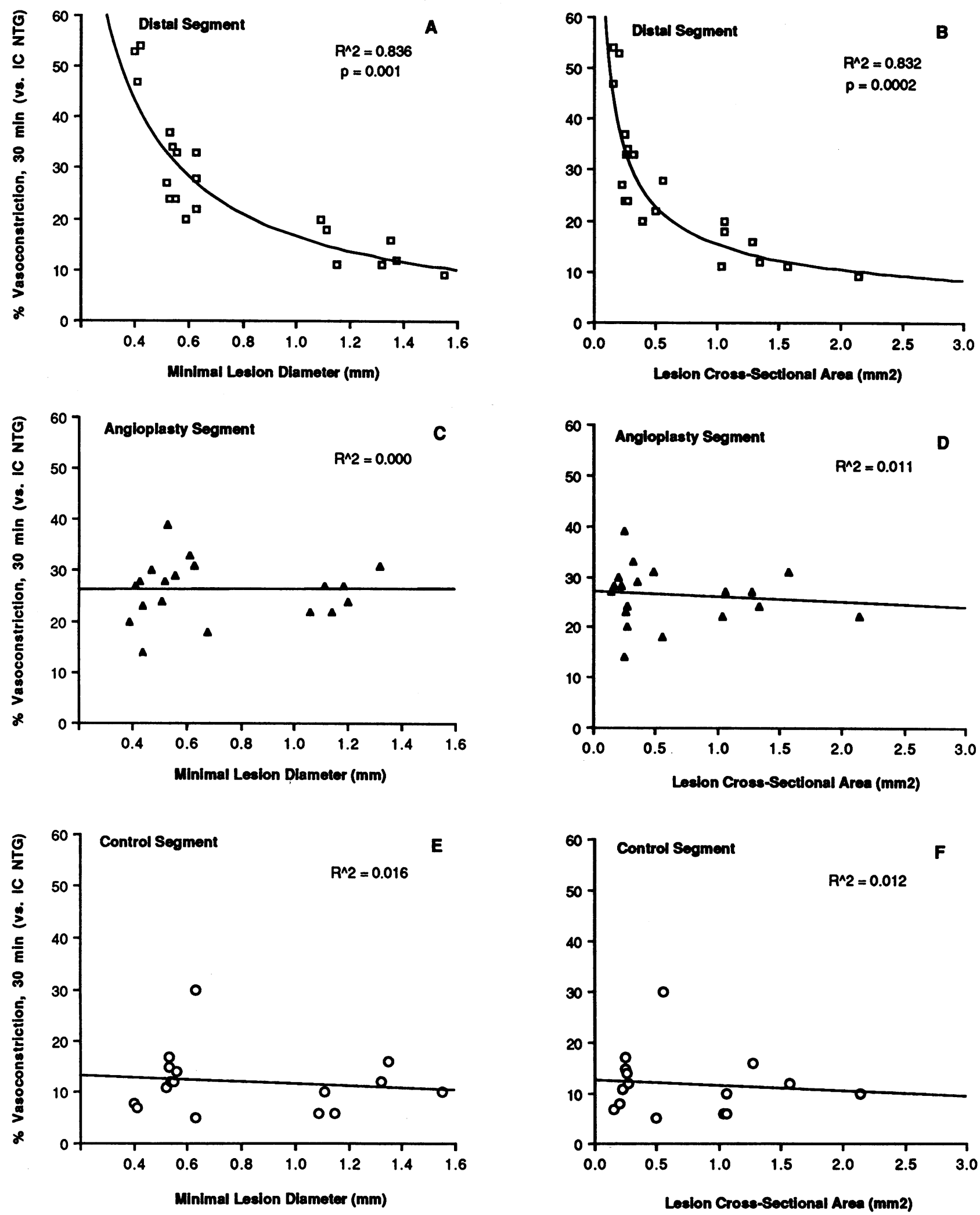

Figure 3. Scattergram plots showing the relationship between lesion severity and the degree of vasoconstriction in the Distal, Angioplasty, and Control segments 30 min after PTCA. $(A-C)$ The relationship between minimal lesion diameter and the severity of vasoconstriction in the Distal $(n=20)$, Angioplasty $(n=20)$, and Control $(n=17)$ segments, respectively. $(B, D$, and $F)$ The relationship between calculated lesion crosssectional area and the severity of vasoconstriction in the Distal $(n=20)$, Angioplasty $(n=20)$, and Control $(n=17)$ segments, respectively. The severity of vasoconstriction in the Distal segment increased exponentially as the minimal lesion diameter and lesion cross-sectional area decreased $\left(R^{2}\right.$ values and $P$ values as shown). In comparison, there was no significant association between lesion severity and the severity of vasoconstriction in the Control or Angioplasty segments $\left(R^{2}\right.$ values as shown). 

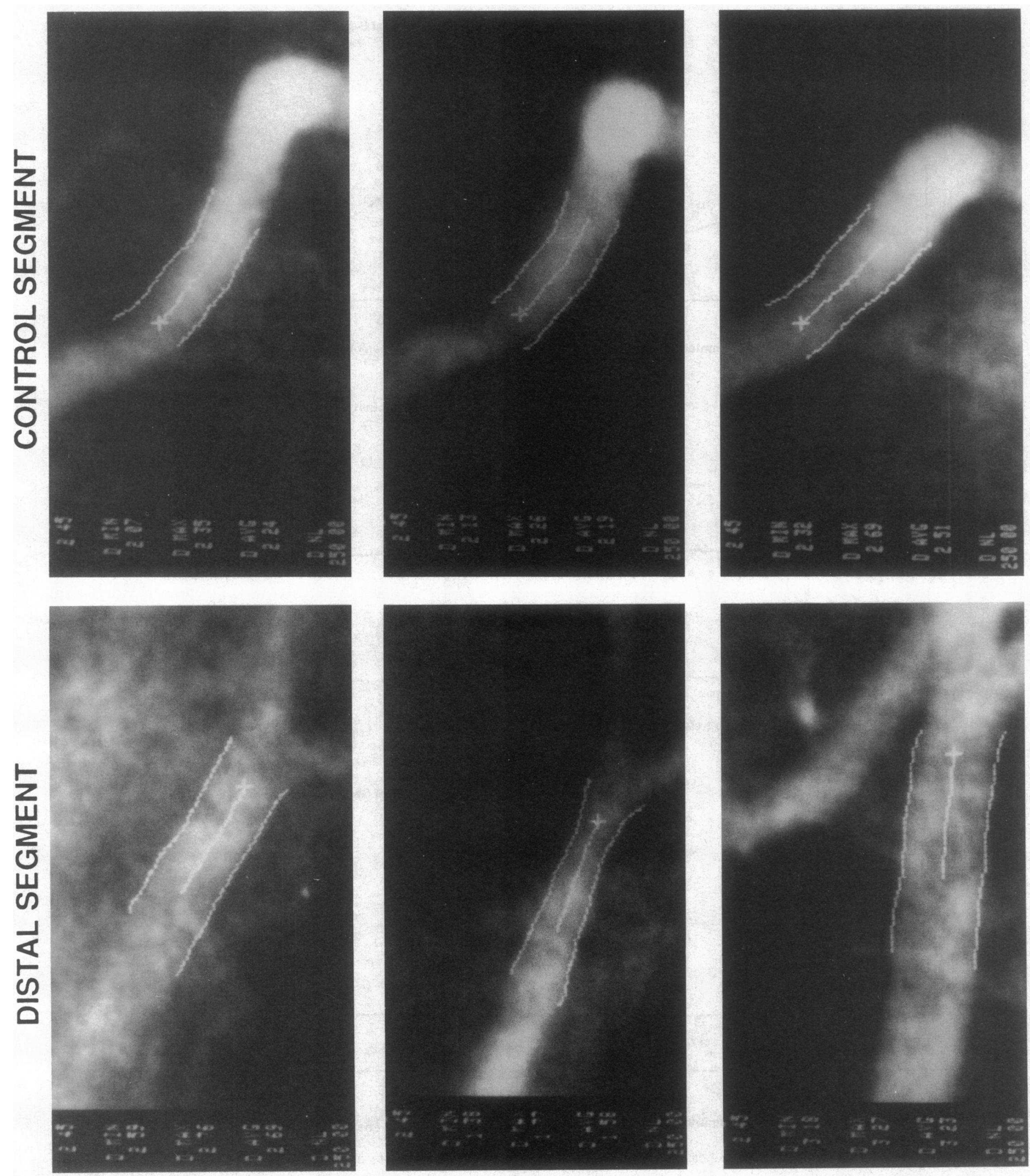

$$
\text { dัํํㄴ }
$$$$
\text { 율 }
$$$$
\stackrel{\frac{1}{ \pm}}{\frac{ \pm}{4}}
$$ 


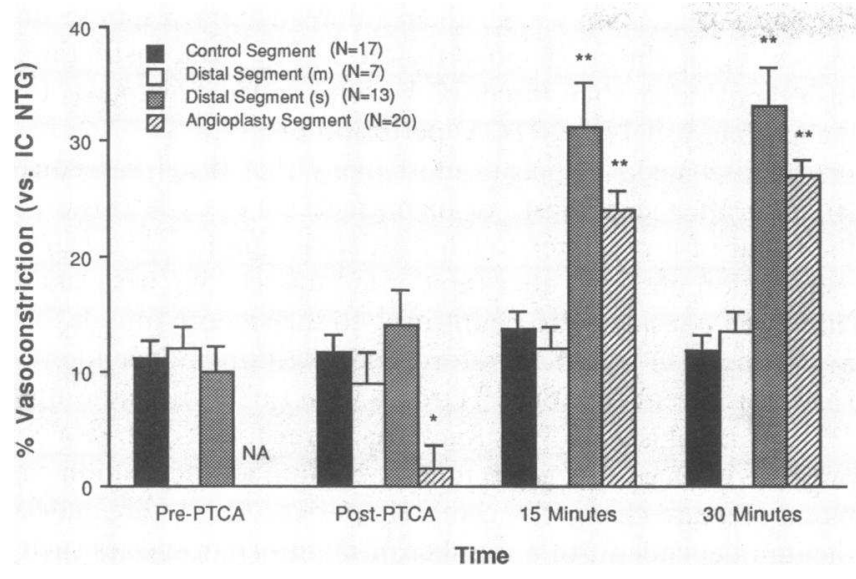

Figure 5. Bar graph showing the time course of spontaneous vasoconstrictor responses after PTCA in the Control $(n=17)$, Distal (see below), and Angioplasty $(n=20)$ segments. Vertical bars represent percent vasoconstriction (mean \pm SEM, see text for definition) for each segment at each time: before PTCA (pre-PTCA), $<5$ min after PTCA (post-PTCA), 15 and 30 min after PTCA. The Distal segment group is divided into two subgroups: those with moderate pre-PTCA lesion severity (Distal segment $(\mathrm{m}), n=7)$, and those with severe or critical lesion severity (Distal segment (s), $n=13$ ). Vasoconstriction in the Distal segment of the severe/critical subgroup was significantly greater at 15 and 30 min after PTCA than post-PTCA, and compared to the vasoconstriction in the Control and Distal (m) segment at 15 and $30 \mathrm{~min}\left({ }^{* *} P s<0.001\right.$ for all comparisons). In contrast, vasoconstriction in the Distal segment (m) subgroup was not significantly changed during the first $30 \mathrm{~min}$ after PTCA and did not differ from the Control segment at any time. The degree of vasoconstriction in the Angioplasty segment at 15 and 30 min after PTCA was significantly greater than that seen in the post-PTCA arteriograms $\left({ }^{* *} P<0.001\right)$. NA, not applicable.

number of stimuli that could alter epicardial coronary artery tone.

First, the distal coronary artery could be exposed to increased concentrations of vasoactive substances, such as serotonin or thromboxane, released from platelets aggregating at the site of balloon injury $(15,16)$. The hypothesis that there was greater vessel trauma after angioplasty of the more severely narrowed segments, leading to increased platelet deposition and greater distal vasoconstriction from platelet-derived vasoactive substances, however, is inconsistent with several observations from this study. One difficulty with this hypothesis is the lack of evidence that there was significantly greater vessel trauma after PTCA of the critical or severe lesions compared to the moderate lesions. The balloon size, ratio of inflated balloon diameter to diameter of adjacent normal artery, total number of balloon inflations, total duration of inflations and post-PTCA incidence of angiographic intimal dissection were not significantly different for the three lesion severity subgroups. A number of studies have demonstrated that the severity of arterial trauma after balloon angioplasty, as judged by the incidence of intimal dissection and acute closure after PTCA (15-17) or determined histologically after in situ angioplasty (18) or after angioplasty in the cholesterol fed rabbit model (19) is not necessarily related to the severity of the lesion being dilated. Severe vessel injury, for example, is often observed after balloon dilatation of moderate lesions (19). If, despite the above considerations, one assumes that there was substantially greater injury and platelet deposition in the more severely narrowed segments, one would expect to see an association between lesion severity and (platelet-mediated) vasoconstriction in the dilated segment paralleling the changes seen in the distal segment. However, there was no correlation between lesion severity and vasoconstriction in the dilated segment. Moreover, if one accepts this platelet-mediator hypothesis, the absence of distal vasoconstriction in patients with moderate lesions would lead to the unlikely conclusion that platelets aggregate minimally or not at all following balloon angioplasty of moderately severe coronary lesions $(20,21)$. Additionally, studies of balloon angioplasty in atherosclerotic rabbits have shown that the potent serotonin antagonist LY53857 inhibits the spasm observed at the site of balloon injury but does not affect the severity of distal vasoconstriction (22), which again suggests that distal vasoconstriction after angioplasty is not platelet mediated. Similarly, the finding that there are no detectable increases in coronary sinus thromboxane concentrations after successful PTCA (23) makes it unlikely that distal vasoconstriction is mediated by in thromboxane. All of these considerations suggest that the significant correlation between pre-PTCA lesion severity and the degree of vasoconstriction observed in the distal segment is not easily explained by the release of platelet-derived vasoactive substances at the site of balloon injury.

Increases in coronary blood flow after successful PTCA may also affect coronary artery tone. In normal epicardial coronary arteries of both dogs $(24,25)$ and humans $(26,27)$, increases in blood flow cause vasodilatation. This flow-mediated vasodilatation appears to be endothelium dependent and may be the result of endothelium-derived relaxing factor(s) (EDRF) release $(26,27)$. In contrast to patients with normal coronary arteries, flow-mediated epicardial coronary artery vasodilatation appears to be blunted or lost in patients with atherosclerotic coronary artery disease (27). Since all of the patients in the current study had coronary artery disease, it is likely that their flow-mediated vasodilatory responses were either blunted or absent. Such a loss of flow-mediated vasodilatation could predispose these subjects to exaggerated increases in perfusion pressure and unopposed vasoconstriction in the setting of increased coronary blood flow after PTCA.

The coronary segment distal to the lesion being dilated may also be exposed to minor trauma from guidewire manipulation, which could theoretically provoke coronary vasocon-

Figure 4. Serial coronary arteriograms demonstrating the vasoconstrictor responses of the Distal and Control segment of a study patient in the critical lesion subgroup (minimum lesion diameter $=0.42 \mathrm{~mm}$, TIMI grade 1 flow prior to PTCA). All images are end-diastolic frames viewed in the same projection and magnification and shown after computerized edge-detection image processing. Images of the Distal segment and the Control segment are shown before PTCA (pre-PTCA), $30 \mathrm{~min}$ after PTCA (30 min), and $3 \mathrm{~min}$ after the administration intracoronary nitroglycerin (after NTG). Marked vasoconstriction is seen in the Distal segment 30 min after PTCA compared to the pre-PTCA diameter with a marked $(56 \%)$ vasodilatory response to nitroglycerin. The Control segment demonstrated modest $(8 \%)$ vasodilatation after nitroglycerin and no spontaneous vasoconstrictor response during the same time period. 
striction via endothelial cell injury with secondary platelet aggregation. Although we cannot totally exclude guidewire trauma as a cause of Distal segment vasoconstriction, this mechanism would not adequately explain the association between pre-PTCA lesion severity and Distal vasoconstriction or the lack of vasoconstriction observed in the Distal segment of the moderate lesion severity subgroup. In addition, the vasoconstriction observed in the coronary artery distal to the dilated lesion was diffuse and not limited to the part of the vessel manipulated by the guidewire (see Fig. 4.).

Finally, depending upon the severity of the lesion being dilated, there may be a rapid increase in perfusion pressure in the epicardial coronary artery distal to the dilated lesion after successful PTCA (28). Although one would expect passive epicardial coronary artery dilatation from an increase in perfusion (distending) pressure after PTCA we observed either no change (moderate lesion subgroup) or a pronounced decrease in coronary diameter (severe and critical lesion severity subgroups) distal to the dilated segment after successful PTCA. Paradoxically, those vessels with presumably the lowest resting perfusion pressure (TIMI grade 1 flow, distal to near-total occlusion) demonstrated the greatest loss of diameter after PTCA, suggesting that hypoperfusion predisposes to vasoconstriction after PTCA (see Fig. 4.). The spontaneous loss of diameter in the distal segment of the severe and critical lesion subgroups cannot be explained by hemodynamic factors, since blood pressure was essentially constant during the serial arteriograms and the Control segment showed no similar loss of diameter. The fact that this progressive loss of diameter cannot be explained by the expected or observed changes in blood pressure, coronary perfusion pressure or coronary blood flow, and was reversed after an intracoronary injection of nitroglycerin suggests that the loss of diameter distal to the dilated lesion was the result of active vasoconstriction.

The magnitude of the increase in perfusion pressure (stretch stimulus) after successful PTCA is directly related to the severity of the resting pressure gradient across the stenosis to be dilated. The relationship between lesion severity and transstenotic pressure gradient across a typical high-grade human coronary stenosis can be approximated by the formula $P_{p} P_{d}=1.8 Q_{s} / d_{\min }{ }^{4}+6.1 Q_{s}^{2} / d_{\min }{ }^{4}$ as described by Brown et al. (8). In this formula $P_{p}-P_{d}$ represents the pressure drop $(\mathrm{mmHg})$ across the stenosis, $Q_{\mathrm{s}}$ the stenosis flow $(\mathrm{ml} / \mathrm{s})$ and $d_{\min }$ the minimal lumen diameter $(\mathrm{mm})$. Assuming a normal range of resting coronary blood flow for medium sized epicardial coronary arteries (1-1.5 ml/s) (29) one can estimate, using this formula, that the average resting transstenotic pressure gradients in our three groups of patients would be 3-6, 25-87, and $110 \rightarrow 200 \mathrm{mmHg}$ for the moderate, severe, and critical lesion subgroups, respectively. It should be recognized, however, that the flow-limiting nature of the most critical lesions may result in an overestimation of the transstenotic pressure gradients when one calculates this gradient based upon normal resting coronary blood flow. The expected increase in perfusion pressure in the distal coronary bed after successful PTCA should be proportional to these resting gradients (28). The actual increase in perfusion pressure after PTCA will also be affected by the degree of collateral blood flow and the adequacy of the PTCA result. Despite these limitations, the observation that the degree of distal vasoconstriction closely parallels the estimated increase in perfusion pressure after successful PTCA provides good evidence that human epicardial coronary arteries may reset their autoregulatory response in the setting of chronic hypoperfusion. Reflex vasoconstriction may then occur when the distal vessel is suddenly exposed to higher than usual (pre-PTCA) perfusion pressure.

As discussed, it appears most likely that distal vasoconstriction after angioplasty of high grade coronary stenoses is the result of a pressure/stretch-mediated stimulus. The vasoconstriction observed after rapid arterial stretching has classically been considered to be intrinsic to the smooth muscle, or myogenic (1-3), and therefore endothelium independent. However, recent studies by Harder (30), Osol (31), Vanhoutte (32-34), and Fischell (35) suggest that some forms of stretch or pressure-induced myogenic tone, including autoregulation to blood pressure changes in cerebral arteries $(30,33)$, are endothelium dependent. In a number of mammalian species such as rats (31), rabbits (35), cats $(30)$, dogs $(33,34)$, and pigs (36) vascular endothelial cells appear capable of functioning as "mechanotransducers" (36), such that a mechanical stimulus (e.g., increased luminal pressure or stretch) may stimulate the release of vasoactive substance(s) (endothelium-derived contractile factor[s]) capable of activating calcium-dependent smooth muscle contraction $(30,33,35,37,38)$. Although speculative, it is possible that the vasoconstriction observed in the distal segment after PTCA of severe and critical stenoses is mediated by such an endothelium-dependent process. We cannot exclude, however, the possibility that the distal vasoconstriction is myogenic.

Implications. The presumptive finding of altered autoregulation as a cause of vasoconstriction in hypoperfused epicardial coronary arteries has several clinical implications. Several investigators have found abnormally depressed coronary flow reserve in $\sim 50 \%$ of patients who undergo successful PTCA (39-41). This depressed coronary blood flow reserve is independent of angiographic or hemodynamic parameters of successful revascularization and eventually normalizes in all patients who do not develop restenosis (39). Wilson et al. speculated that prolonged hypoperfusion distal to an epicardial coronary stenosis may "[alter] the ability of the arteriolar vasculature to autoregulate in response to a sudden restoration of normal perfusion pressure" (39). If the alteration in coronary autoregulation that we observed in epicardial vessels were generalized to the coronary arterioles, this might explain the finding of transient depression of coronary flow reserve after successful PTCA.

A second important clinical finding of this study is the ability of intracoronary nitroglycerin to reverse this coronary vasoconstriction. We have previously reported that intravenous nitroglycerin administered at doses that reduce the systolic blood pressure by $\sim 20 \mathrm{mmHg}$ is capable of preventing vasospasm in both the Distal and Angioplasty segments (7). Based on these observations and the findings that orally administered calcium channel blockers and aspirin do not prevent vasoconstriction in the Angioplasty or Distal segments, we routinely administer relatively high-dose systemic nitroglycerin therapy during, and for at least $24 \mathrm{~h}$ after PTCA.

Finally, in extreme cases this vasospasm may provoke ischemia and/or myocardial infarction after PTCA or coronary artery bypass grafting surgery (6). In one particularly illustrative case occlusive coronary artery spasm leading to myocardial infarction occurred after bypass grafting of a chronically occluded left anterior descending coronary artery, with no evidence of spasm in a grafted obtuse marginal coronary artery 
with a moderate (60\%) stenosis. Myocardial infarction secondary to severe coronary artery spasm after coronary bypass grafting of chronically hypoperfused vessels could explain, in part, the intriguing observation that a majority of postoperative myocardial infarctions are associated with widely patent bypass grafts at autopsy or arteriography $(42,43)$.

Limitations of the study. Despite the care taken to control for confounding clinical variables there are several potential limitations of this study. It is recognized that collateral blood flow may increase the coronary perfusion pressure distal to severe or critical stenoses. To minimize the effects of well-developed coronary collaterals on the transstenotic pressure drop we excluded from entry patients with well-developed collaterals supplying the vessel to be dilated ( $>$ grade 2 collaterals) (12).

Ideally, transstenotic pressure gradients should have been used to correlate alterations in epicardial autoregulation with chronic hypoperfusion. Although these pressure gradients can be measured using balloon catheters before and after PTCA, these measurements are often inaccurate and may be erroneously affected by vessel tortuosity, balloon catheter size, and changes in coronary blood flow $(28,44)$. For these reasons we elected to use primarily angiographic measures of lesion severity to assess the relationship between hypoperfusion and distal vasoconstriction after PTCA. Finally, our methodolology does not allow us to comment upon the latency period required to reset or readjust autoregulation in the setting of hypoperfusion, nor the duration of altered autoregulation after the restoration of normal coronary blood flow.

Summary. The severity of coronary artery vasoconstriction distal to the site of successful PTCA is closely correlated with lesion severity before PTCA. This distal vasoconstriction begins shortly after successful revascularization and appears to peak at 15-30 min after PTCA. These observations are consistent with the hypothesis that human epicardial coronary arteries can reset their autoregulatory responsiveness in the setting of chronic hypoperfusion such that restoration of more normal levels of perfusion pressure after successful PTCA may provoke reflex vasoconstriction. It is likely that this vasospasm is mediated by either an endothelium-dependent or myogenic mechanism. These findings have important physiologic implications concerning the dynamics and plasticity of human coronary artery blood flow regulation as well as clinical implications regarding the etiology and prophylaxis of coronary artery spasm after PTCA and coronary artery bypass graft surgery.

\section{Acknowledgments}

We thank Dr. David Clark and Dr. Marc Gradman for their assistance in patient recruitment and participation in the study protocol, and Robert Kernoff for his assistance with photography.

Dr. Fischell is a Clinical Investigator of the National Heart, Lung, and Blood Institute (grant HL-02001-01). This paper was accepted for finalist presentation, Young Investigators Award Competition, American College of Cardiology, 19 March 1990.

\section{References}

1. Bayliss, W. M. 1902. On the local reactions of the arterial wall to changes of internal pressure. J. Physiol. (Lond.). 28:220-231.

2. Koch, A. R. 1964. Some mathematical forms of autoregulating models. Circ. Res. 15(Suppl. 1):269-279.
3. Bevan, J. A. 1985. Vascular myogenic or stretch-dependent tone. J. Cardiovasc. Pharm. 7(Suppl. 3):S129-136.

4. Vatner, S. F., M. Pagani, W. T. Manders, and A. D. Pasipoularides. 1979. Alpha adrenergic vasoconstriction and nitroglycerin vasodilation of large coronary arteries in the conscious dog. J. Clin. Invest. 45:654-660.

5. Hodgson, J. M., M. D. Cohen, S. Szenpetery, and M. D. Thames. 1989. Effects of regional alpha- and beta-blockade on resting and hyperemic coronary blood flow in conscious, unstressed humans. Circulation. 79(4):797-809.

6. Fischell, T. A., T. V. McDonald, M. T. Grattan, D. C. Mille, and M. L. Stadius. 1989. Occlusive coronary-artery spasm as a cause of acute myocardial infarction after coronary-artery bypass grafting. $N$. Engl. J. Med. 320(6):400-401.

7. Fischell, T. A., G. Derby, T. M. Tse, and M. L. Stadius. 1988. Coronary artery vasoconstriction routinely occurs after percutaneous transluminal coronary angioplasty: a quantitative arteriographic analysis. Circulation. 78(6):1323-1334.

8. Brown, B. G., E. L. Bolson, and H. T. Dodge. 1984. Dynamic mechanisms in human coronary stenosis. 1984. Circulation. 70(6):917-922.

9. Rentrop, K. P., M. Cohen, H. Blanke, and R. A. Phillips. 1985. Changes in collateral channel filling immediately after controlled coronary artery occlusion by an angioplasty balloon in human subjects. $J$. Am. Coll. Cardiol. 5:587-591.

10. Fujita, M., S. Sasayama, H. Asanoi, H. Nakajima, O. Sakai, and A. Ohno. 1988. Improvement of treadmill capacity and collateral circulation as a result of exercise with heparin pretreatment in patients with effort angina. Circulation. 77(5):1022-1029.

11. Passamani, E., and the TIMI Study Group. 1985. The thrombolysis in myocardial infarction (TIMI) trial. N. Engl. J. Med. 312(14):932-936.

12. Alderman, E. L., L. E. Berte, D. C. Harrison, and W. Sanders. 1981. Quantitation of coronary artery dimensions using digital image processing. Diag. Radiol. 314:273-277.

13. McMahon, M. M., B. G. Brown, R. Cukingnan, E. L. Rolett, E. Bolson, M. Frimer, and H. T. Dodge. 1979. Quantitative coronary angiography: measurement of the "critical" stenosis in patients with unstable angina and single-vessel disease without collaterals. Circulation. 60:106-113.

14. Sasaguri, T., T. Itoh, M. Hirata, K. Kitamura, and H. Kuriyama. 1987. Regulation of coronary artery tone in relation to the activation of signal transductors that regulate calcium homeostasis. $J$ Am Coll Cardiol. 9:1167-1175.

15. Ischinger, T., A. R. Gruentzig, B. Meier, and K. Galan. 1986. Coronary dissection and total coronary occlusion associated with percutaneous transluminal coronary angioplasty: significance of initial angiographic morphology of coronary stenoses. Circulation. 74:13711378.

16. Ellis, S. G., G. S. Roubin, S. B. King, J. S. Douglas, W. S. Weintraub, R. G. Thomas, and W. R. Cox. 1988. Angiographic and clinical predictors of acute closure after native vessel coronary angioplasty. Circulation. 77:372-379.

17. Nichols, A. B., R. Smith, A. D. Berke, R. A. Shlofmitz, and E. R. Powers. 1989. Importance of balloon size in coronary angioplasty. J. Am. Coll. Cardiol. 13:1094-1100.

18. Lyon, R. T., C. K. Zarins, C. T. Lu, C. F. Yang, and S. Glagov. 1987. Vessel, plaque, and lumen morphology after transluminal balloon angioplasty. Arteriosclerosis. 7:306-314.

19. Sanborn, T. A., D. P. Faxon, C. Haudenschild, S. B. Gottsman, and T. J. Ryan. 1983. The mechanism of transluminal angioplasty: evidence for formation of aneurysms in experimental atherosclerosis. Circulation. 68:1136-1140.

20. Lam, J. Y., J. H. Chesebro, P. M. Steele, L. Badimon, and V. Fuster. 1987. Is vasospasm related to platelet deposition? Relationship in a porcine preparation of arterial injury in vivo. Circulation. 75:243-248. 
21. Lam, J. Y. T., J. H. Cheseboro, and V. Fuster. 1988. Platelets, vasoconstriction, and nitroglycerin during arterial wall injury: a new antithrombolytic role for an old drug. Circulation. 78:712-716.

22. Sigal, S. L., I. J. Sarembock, P. J. LaVeau, T. L. Yang, and M. D. Ezekowitz. 1988. A specific serotonin receptor antagonist prevents proximal but not distal spasm following balloon angioplasty. Circulation. (Suppl. II):I-270.

23. Peterson, M. B., V. Machaj, P. C. Block, I. Palacios, D. Philbin, and W. D. Watkins. 1986. Thromboxane release during percutaneous transluminal coronary angioplasty. Am. Heart J. 111(1);1-6.

24. Inoue, T., H. Tomoike, K. Hisano, and M. Nakamura. 1988. Endothelium determines flow-dependent dilation of the epicardial coronary artery in dogs. J. Am. Col. Cardiol. 11:187-191.

25. Hintze, T. H., and S. F. Vatner. 1984. Reactive dilation of large coronary arteries in conscious dogs. Circ. Res. 54:50-57.

26. Drexler, H., A. Zeiher, H. Wollschlager, and T. Bonzel. 1988. Flow-dependent coronary dilation in man. Circulation. (Suppl. II):II171.

27. Cox, D. A., J. A. Vita, C. B. Treasure, R. D. Fish, R. W Alexander, P. Ganz, and A. P. Selwyn. 1989. Atherosclerosis impairs, flow-mediated dilation of coronary arteries in humans. Clin. Res. $80: 458-465$.

28. Serruys, P. W., W. Wijns, J. H. C. Reiber, P. de Feyter, M. Van den Brand, F. Piscione, and P. G. Hugenholtz. 1985. Values and limitations of transstenotic pressure gradients measured during percutaneous transluminal coronary angioplasty. Herz. 10(6):337-342.

29. Ganz, W., K. Kamura, H. S. Marcus, S. Donoso, S. Yashida, and H. J. C. Swan. 1971. Measurements of coronary sinus blood flow by continuous thermodilution in man. Circulation. 44:181-195.

30. Harder, D. R. 1987. Pressure-induced myogenic activation of cat cerebral arteries is dependent on intact endothelium. Circ. Res. 60:102-107.

31. Osol, G., R. Osol, and W. Halpern. 1987. Pressure-dependent modulation of myogenic tone by cerebral endothelium (abstract). Circulation. 76(Suppl. IV):IV-382.

32. Vanhoutte, P. M. 1987. Endothelium-dependent contractions in arteries and veins. Blood Vessels. 24:141-144.

33. Katusic, Z. S., J. T. Shepherd, and P. M. Vanhoutte. 1987.
Endothelium-dependent contraction to stretch in canine basilar arteries. Am. J. Physiol. 252:H671-673.

34. Katusic, Z. S., J. T. Shepherd, and P. M. Vanhoutte. 1986. Endothelium-dependent contraction to stretch in canine basilar arteries (abstract). Fed. Proc. 45:289.

35. Fischell, T. A., U. Nellessen, D. E. Johnson, and R. Ginsburg. 1989. Endothelium-dependent arterial vasoconstriction after balloon angioplasty. Circulation. 79:899-910.

36. Lansman, J. B., T. J. Hallam, and T. J. Rink. 1987. Single stretch-activated ion channels in vascular endothelial cells as mechanotransducers. Nature (Lond.). 325:811-813.

37. Hickey, K. A., G. Rubanyi, R. J. Paul, and R. F. Highsmith. 1985. Characterization of a coronary vasoconstrictor produced by cultured endothelial cells. Am. J. Physiol. 248:C550-556.

38. Gillespie, M. N., J. O. Owasoyo, I. F. McMurty, and R. F. O'Brien. 1986. Sustained coronary vasoconstriction provoked by a peptidergic substance released from endothelial cells in culture. $J$. Pharmacol. Exp. Ther. 236:339-343.

39. Wilson, R. F., M. R. Johnson, M. L. Marcus, P. E. Aylward, D. J. Skorton, S. Collins, and C. W. White. 1988. The effect of coronary angioplasty on coronary flow reserve. Circulation. 77:873-885.

40. Hodgson, J. M., R. S. Riley, A. S. Most, and D. O. Williams. 1987. Assessment of coronary flow reserve using digital angiography before and after successful percutaneous transluminal angioplasty. Am. J. Cardiol. 60:61-65.

41. Bates, E. R., F. M. Aueron, V. Legrand, M. T. Le Free, G. B. Mancini, J. M. Hodgson, and R. A. Vogel. 1985. Comparative longterm effects of coronary artery bypass graft surgery and percutaneous transluminal angioplasty on regional coronary flow reserve. Circulation. 72:833-842.

42. Lemmer, J. H., and M. M. Kirsh. 1988. Coronary artery spasm following coronary artery surgery. Ann. Thorac. Surg. 46:108-115.

43. Bulkley, B. H., and G, M. Hutchins. 1977. Myocardial consequences of coronary artery bypass graft surgery: the paradox of necrosis in areas of revascularization. Circulation. 56:906-913.

44. Feldman, R. C., and D. J. Anderson. 1985. Gradients at PTCA physiological or artifactual? J. Am. Coll. Cardiol. 5:525. 